cipitate of cobaltous sulphide proves the presence of the acetate ion. ${ }^{1}$

In the absence of the cyanide, chromate, chlorate and iodide ions the well-known test for acetate with concentrated sulphuric acid and alcohol (formation of ethyl acetate) may also be used.

$\mathrm{SiO}_{3}{ }^{\prime \prime}$.-Acidify a portion of the solution with hydrochloric acid, evaporate to dryness, gently heat the residue, then treat with dilute hydrochloric acid and afterwards with hot water. An insoluble residue indicates the silicate ion. Confirm by the usual bead tests, viz., skeleton in metaphosphate, transparency in sodium carbonate bead, or by treatment with hydrofluoric acid.

As. O $_{4}^{\prime \prime \prime}$-- (To be used in case the arsenite ion has been found.) If arsenite ion has been found in Group III, the appearance of arsenate ion in Group VII does not prove the presence of that ion in the original solution (see note on Group V). To test for the arsenate ion in such an instance, to a portion of Solution $\mathrm{A}$ add magnesia mixture as long as a precipitate forms, filter out and wash the precipitate, dissolve it in concentrated hydrochloric acid, add a little sulphurous acid and boil until the excess is completely expelled. Pass in hydrogen sulphide. A yellow precipitate of arsenious sulphide indicates the arsenate ion.

Grovp X.- $\mathrm{CO}_{3}{ }^{\prime \prime}$.

Acidify the original solution or substance with dilute hydrochloric acid and test the escaping gas with a film of lime-water.

\title{
SOME NEW FORMS OF LECTURE AND LABORATORY APPARATUS.
}

By G. B. Frankforter aXd Frarcis C. FRART.

Received April 10, 1905.

THE following pieces of apparatus will, we believe, be of some little interest to those who are teachers of chemistry. These forms of apparatus have been used both on the lecture table and in the laboratory, and have given such satisfactory results that we venture to give them a brief notice.

One of the essentials in both lecture and laboratory apparatus is simplicity in construction and manipulation. If a piece of apparatus will give good results in the hands of the average student,

1 Beredict: Am. Chem. J., 32, 481 (1904). 
it may be considered as having some merit. If it does not, it will certainly have a short life. The apparatus described below has given good results in this laboratory, and we hope that it may prove useful to others.

Apparatus for the Determination of the Hydrogen Equivalent of Zinc.-Various pieces of apparatus have been used on the lecture table and in the laboratory for determining the hydrogen equivalent of zinc, but none which we have tried proved free from faults, especially in the hands of the students. The apparatus described below, will, we believe, eliminate the chief sources of error in this determination.

As shown in Fig. I, it consists of an Erlenmeyer flask A of about $200 \mathrm{cc}$., fitted with a double-bored cork. Through one hole passes a funnel tube bent in the form of an $S$, and with the funnel part extended into a wide tube. The shape of the funnel tube is the only peculiar part of the apparatus. By using a wide tube, so that the liquid always stands in the wide part, acid may be introduced without any danger of carrying air bubbles into the flask. The zinc is placed in the flask, the whole apparatus is filled with freshly boiled water, and sulphuric acid (roocc. of acid I :4) is poured into the funnel tube. The hydrogen liberated is collected in a measuring tube over water. The last trace of gas is driven out of the apparatus by pouring water into the funnel tube. Calculations are made in the usual manner.

APPARATUS FOR MAKING SULPHURIC ACID.

The old apparatus, described in nearly all the large text-books, must be admitted by every one to be unsatisfactory for laboratory use. For instance, it is not satisfactory to make sulphur dioxide from sulphuric acid and copper, for the student will immediately see that you are making sulphuric acid from sulphuric acid, instead of sulphur or pyrites. The apparatus described below, we believe to be an improvement upon the old form both in construction and manipulation.

The apparatus, Figs. 2 and 4 , is clamped in such a position that tubes $A$ and $E$ are almost horizontal, $A$ being slightly higher than $\mathrm{E}$. Tube $\mathrm{C}$ is connected with a flask of boiling water, provided with a safety tube, for supplying steam under pressure, and tube $D$ with a nitric oxide generator. $E$ is connected with a Liebig condenser by means of a short piece of rubber tubing. The other 
end of the condenser projects into a large bottle or flask. As soon as steam and nitric oxide are passing through the apparatus, a porcelain boat full of burning sulphur is introduced into $A$, and the process begins. Enough acid for experimental purposes may be made in a few minutes. The apparatus can be made by any one with a moderate knowledge of glass-blowing, and when made

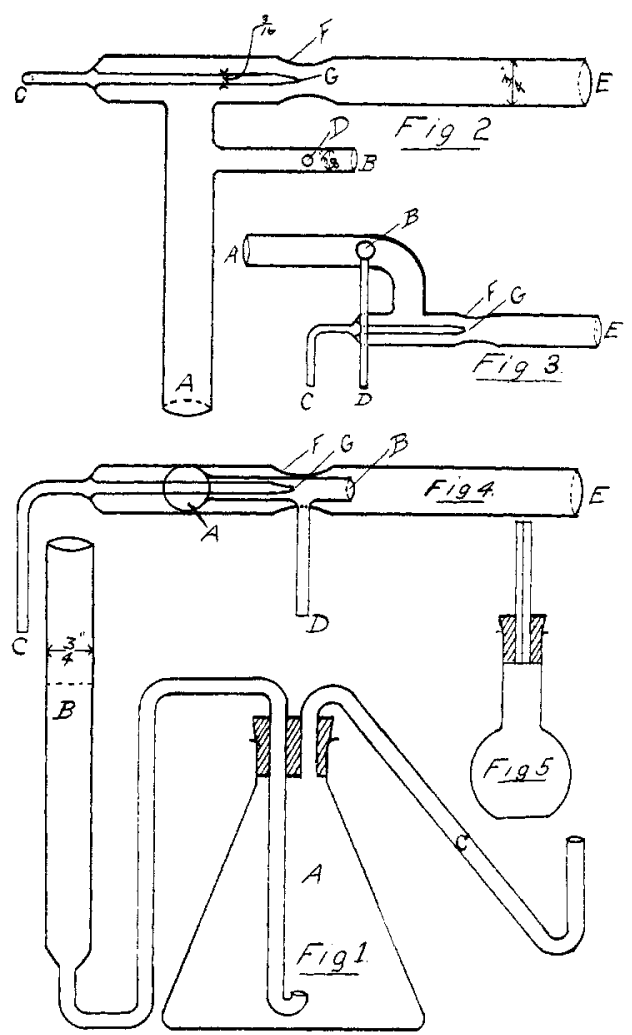

of good glass there is little danger of breakage. Fig. 3 represents the apparatus used for lecture work. The apparatus is best used under a hood, but may be used on any table by placing a beaker containing a little ammonia near the neck of the large bottle or flask, so as to absorb the oxides which escape.

CHARLES' LAW.

The apparatus used in proving Charles' Law consists of a small, 
flat-bottomed flask of about Ioo cc. The flask is fitted with a single-bored cork, through which passes a fine, capillary tube about 4 inches long. The cork is pressed into the neck of the flask as far as it will go, and the capacity of the flask determined. The position of the cork is marked by a scratch on the neck of the flask.

The flask is thoroughly dried, and the cork containing the capillary tube is pressed into the neck until the lower end is even with the scratch. Using the capillary tube as a handle, the flask is immersed in a bath of boiling water. It is held in this position until the air in the flask has reached the temperature of the water. The temperature is then taken. The finger is moistened and placed over the end of the capillary tube, the apparatus is transferred to a bath of cold water, capillary end first, and the finger removed. As the apparatus cools, the water enters the flask, the quantity of water measuring the change in the volume of the air. From the data thus obtained, the law may be verified.

Care must be taken to handle the apparatus by means of the capillary tube so as to avoid the error due to the heat of the hand. The chief cause of error is that due to imperfect drying of the apparatus, especially the cork, capillary and the space between them.

TNIVERSITY OF MINNESOTA,

MINNEAPOLIS, MINN.

[CONTRIBUTIONS FROM THE RESEARCH LABORATORY OF PHYSICAL, ChEMISTRy OF THE MASSAChuset's INSTITUTE OF TEChNOLOGY, No. 7.]

\section{THE SOLUBILITY OF LEAD SULPHATE IN AMMONIUM ACETATE SOLUTIONS.}

By Arthur A. Noyes and William H. Whitcomb.

Received April 20, I905.

I. HYPOTHESES AS TO THE CAUSE OF THE SOLUBILITYINCREASE.

IT is A well-known fact, and one often made use of in chemical analysis, that lead sulphate, though difficultly soluble in water, dissolves in considerable quantity in a strong ammonium acetate solution. As to the magnitude of this solubility we have, however, been able to find only two quantitative statements, that of Long, ${ }^{1}$ and that of Bischof, ${ }^{2}$ both of which relate to very concentrated solutions of ammonium acetate.

1 Am. Chem.J., 22, 217 (1899).

2 Comey's "Dictionary of Solubilities." 